CUBO A Mathematical Journal

Vol.14, No 03 , (129-142). October 2012

\title{
Existence of deviating fractional differential equation
}

\author{
RABHA W. IBRAHIM \\ Institute of Mathematical Sciences \\ University Malaya, 50603 \\ Kuala Lumpur, Malaysia \\ email: rabhaibrahim@yahoo.com
}

\begin{abstract}
In this paper we shall establish sufficient conditions for the existence of solutions of a class of fractional differential equation (Cauchy type ) and its solvability in a subset of the Banach space. The main tool used in our study is the non-expansive operator technique. The non integer case is taken in sense of Riemann-Liouville fractional operators. Applications are illustrated.
\end{abstract}

\section{RESUMEN}

En este artículo establecemos condiciones suficientes para la existencia de soluciones de una clase de ecuaciones diferenciales fraccionales (del tipo Cauchy) y su solubilidad en un subconjunto de un espacio de Banach. La principal herramienta utilizada en nuestro estudio es la técnica del operador no expansivo. El caso no entero se escoge en el sentido de operadores fraccionales Riemann-Liouville. Además, se ilustran aplicaciones.

Keywords and Phrases: Fractional calculus; fractional differential equation; Cauchy equation; Riemann-Liouville fractional operators; Volterra integral equation; non-expansive mapping; iterative differential equation

2010 AMS Mathematics Subject Classification: 34A12. 


\section{$1 \quad$ Introduction}

Fractional calculus and its applications (that is the theory of derivatives and integrals of any arbitrary real or complex order) has importance in several widely diverse areas of mathematical physical and engineering sciences. It generalized the ideas of integer order differentiation and nfold integration. Fractional derivatives introduce an excellent instrument for the description of general properties of various materials and processes. This is the main advantage of fractional derivatives in comparison with classical integer-order models, in which such effects are in fact neglected. The advantages of fractional derivatives become apparent in modeling mechanical and electrical properties of real materials, as well as in the description of properties of gases, liquids and rocks, and in many other fields [1-5].

The class of fractional differential equations of various types plays important roles and tools not only in mathematics but also in physics, control systems, dynamical systems and engineering to create the mathematical modeling of many physical phenomena. Naturally, such equations required to be solved. Many studies on fractional calculus and fractional differential equations, involving different operators such as Riemann-Liouville operators, Erdlyi-Kober operators, WeylRiesz operators, Caputo operators and Grnwald-Letnikov operators, have appeared during the past three decades. The existence of positive solution and multi-positive solutions for nonlinear fractional differential equation are established and studied [6-8]. Moreover, by using the concepts of the subordination and superordination of analytic functions, the existence of analytic solutions for fractional differential equations in complex domain are suggested and posed in $[9,10]$.

Our aim in this paper is to consider the existence and uniqueness of nonlinear Cauchy problems of fractional order in sense of Riemann-Liouville operators. Also, two theorems in the analytic continuation of solutions are studied. In the fractional Cauchy problems, we replace the first order time derivative by a fractional derivative. Fractional Cauchy problems are useful in physics. Recently, the author studied the the fractional Cauchy problems in complex domain [11].

One of the most frequently used tools in the theory of fractional calculus is furnished by the Riemann-Liouville operators (see[6-8]). The Riemann-Liouville fractional derivative could hardly pose the physical interpretation of the initial conditions required for the initial value problems involving fractional differential equations. Moreover, this operator possesses advantages of fast convergence, higher stability and higher accuracy to derive different types of numerical algorithms.

Definition 1.1. The fractional (arbitrary) order integral of the function $f$ of order $\alpha>0$ is defined by

$$
I_{a}^{\alpha} f(t)=\int_{a}^{t} \frac{(t-\tau)^{\alpha-1}}{\Gamma(\alpha)} f(\tau) d \tau
$$


When $a=0$, we write $I_{a}^{\alpha} f(t)=f(t) * \phi_{\alpha}(t)$, where $(*)$ denoted the convolution product (see [7]), $\phi_{\alpha}(\mathrm{t})=\frac{\mathrm{t}^{\alpha-1}}{\Gamma(\alpha)}, \mathrm{t}>0$ and $\phi_{\alpha}(\mathrm{t})=0, \mathrm{t} \leq 0$ and $\phi_{\alpha} \rightarrow \delta(\mathrm{t})$ as $\alpha \rightarrow 0$ where $\delta(\mathrm{t})$ is the delta function.

Definition 1.2. The fractional (arbitrary) order derivative of the function $f$ of order $0 \leq \alpha<1$ is defined by

$$
D_{a}^{\alpha} f(t)=\frac{d}{d t} \int_{a}^{t} \frac{(t-\tau)^{-\alpha}}{\Gamma(1-\alpha)} f(\tau) d \tau=\frac{d}{d t} I_{a}^{1-\alpha} f(t)
$$

Remark 1.1. From Definition 1.1 and Definition 1.2, we have

$$
\mathrm{D}^{\alpha} \mathrm{t}^{\mu}=\frac{\Gamma(\mu+1)}{\Gamma(\mu-\alpha+1)} \mathrm{t}^{\mu-\alpha}, \mu>-1 ; 0<\alpha<1
$$

and

$$
\mathrm{I}^{\alpha} \mathrm{t}^{\mu}=\frac{\Gamma(\mu+1)}{\Gamma(\mu+\alpha+1)} \mathrm{t}^{\mu+\alpha}, \mu>-1 ; \alpha>0
$$

\section{Preliminaries}

We extract here the basic theory of non-expansive mappings in order to offer the notions and results that will be needed in the next sections of the paper. Let $(X, d)$ be a metric space. A mapping $P: X \rightarrow X$ is said to be an $v$-contraction if there exists $v \in[0,1)$ such that

$$
\mathrm{d}(\mathrm{Px}, \mathrm{Py}) \leq v \mathrm{~d}(\mathrm{x}, \mathrm{y}), \quad \forall x, y \in X
$$

In the case where $v=1$ the mapping $P$ is said to be non expansive. Let $K$ be a nonempty subset of a real normed linear space $\mathrm{E}$ and $\mathrm{P}: \mathrm{K} \rightarrow \mathrm{K}$ be a map. In this setting, $\mathrm{P}$ is non-expansive if

$$
\|P x-P y\| \leq\|x-y\| \quad \forall x, y \in K .
$$

The following result is a fixed point theorem for non expansive mappings, due to Browder, Ghode and Kirk, see e.g. [12]:

Theorem 2.1. Let $K$ be a nonempty closed convex and bounded subset of a uniformly Banach space $\mathrm{E}$. Then any non expansive mapping $\mathrm{P}: \mathrm{K} \rightarrow \mathrm{K}$ has at least a fixed point.

Definition 2.1. Let $K$ be a convex subset of a normed linear space $E$ and let $P: K \rightarrow K$ be a self-mapping. Given an $x_{0} \in K$ and a real number $\lambda \in[0,1]$, the sequence $x_{n}$ defined by the formula

$$
x_{n+1}=(1-\lambda) x_{n}+\lambda P x_{n}, \quad n=0,1,2, \ldots
$$

is usually called Krasnoselskij iteration or Krasnoselskij-Mann iteration. 
Definition 2.2. Let $K$ be a convex subset of a normed linear space $E$ and let $P: K \rightarrow K$ be a self-mapping. Given an $x_{0} \in K$ and a real number $\lambda_{n} \in[0,1]$, the sequence $x_{n}$ defined by the formula

$$
x_{n+1}=\left(1-\lambda_{n}\right) x_{n}+\lambda_{n} P x_{n}, \quad n=0,1,2, \ldots
$$

is usually called Mann iteration.

Edelstein [13] proved that strict convexity of E suffices for the Krasnoselskij iteration converge to a fixed point of P. While, Egri and Rus [14] proved that for any subset of E, the Mann iteration converge to a fixed point of $\mathrm{P}$ when $\mathrm{P}$ is a non-expansive mapping.

We need the following results, which can be found in [15]:

Lemma 2.1. Let $\mathrm{K}$ be a convex and compact subset of a Banach space $\mathrm{E}$ and let $\mathrm{P}: \mathrm{K} \rightarrow \mathrm{K}$ be a non-expansive mapping. If the Mann iteration process $x_{n}$ satisfies the assumptions

(a) $x_{n} \in K$ for all positive integers $n$,

(b) $0 \leq \lambda_{n} \leq \mathrm{b}<1$ for all positive integers $n$,

(c) $\sum_{n=0}^{\infty} \lambda_{n}=\infty$.

Then $x_{n}$ converges strongly to a fixed point of $P$.

Lemma 2.2. Let $K$ be a closed bounded convex subset of a real normed space $E$ and $P: K \rightarrow K$ be a non-expansive mapping. If $\mathrm{I}-\mathrm{P}$ maps closed bounded subset of $\mathrm{E}$ into closed subset of $\mathrm{E}$ and $x_{n}$ is the Mann iteration, with $\lambda_{n}$ satisfying assumptions (a)-(c) in Lemma 2.1, then $x_{n}$ converges strongly to a fixed point of $\mathrm{P}$ in $\mathrm{K}$.

\section{Existence theorems and approximation of solutions}

For most of the differential and integral equations with deviating arguments that appear in recent literature, the deviation of the argument usually involves only the time itself. However, another case, in which the deviating arguments depend on both the state variable $u$ and the time $t$, is of importance in theory and practice. Equations of the form

$$
\mathrm{u}^{\prime}(\mathrm{t})=\mathrm{f}(\mathrm{t}, \mathrm{u}(\mathrm{u}(\mathrm{t})))
$$

are called iterative differential equations. These equations are important in the study of infection models and are related to the study of the motion of charged particles with retarded interaction (see [16-18]).

In this section, we establish the existence and uniqueness results for the fractional differential equation

$$
D^{\alpha} u(t)=f(t, u(t), u(u(t)))
$$


with initial condition $\mathfrak{u}(0)=\mathfrak{u}_{0}$, where $t, \mathfrak{u}_{0} \in J:=[0, T]$ and $f \in C(J \times J \times J, J)$. For $t \in J$ denote

$$
M_{t}=\max \{t, T-t\}
$$

and

$$
C_{L, \alpha}=\left\{u:\left|u\left(t_{1}\right)-u\left(t_{2}\right)\right| \leq \frac{L}{\Gamma(\alpha+1)}\left|t_{1}-t_{2}\right|^{\alpha}, \forall t_{1}, t_{2} \in J\right\}, \quad L>0 .
$$

It is clear that $C_{L, \alpha}$ is a nonempty convex and compact subset of the Banach space $(C[J],\|\|$.$) ,$ where $\|x\|=\sup _{t \in J}|x(t)|$.

Theorem 3.1. Assume that the following conditions are satisfied for the initial value problem (1):

(A1) $f \in C[J \times J \times J, J]$;

(A2) $\exists \ell>0:\left|f\left(t, u_{1}, u_{2}\right)-f\left(t, v_{1}, v_{2}\right)\right| \leq \ell\left[\left|u_{1}-v_{1}\right|+\left|u_{2}-v_{2}\right|\right], \forall t, u_{i}, v_{i}, i=1,2 \in J$;

(A3) If $L$ is the Lipschitz constant such that $\left|u\left(t_{1}\right)-u\left(t_{2}\right)\right| \leq \frac{L}{\Gamma(\alpha+1)}\left|t_{1}-t_{2}\right|^{\alpha}$, then

$$
M=\max \{|f(t, u, v)|:(t, u, v) \in J \times J \times J\} \leq \frac{L}{2}
$$

(A4) One of the following conditions holds:

(a) $M \frac{T^{\alpha}}{\Gamma(\alpha+1)} \leq M_{\mathfrak{u}_{0}}$, where $M_{\mathfrak{u}_{0}}=\max \left\{\mathfrak{u}_{0}, T-\mathfrak{u}_{0}\right\}$;

(b) $u_{0}=0, M \frac{T^{\alpha}}{\Gamma(\alpha+1)} \leq T-u_{0}, f(t, u, v) \geq 0, \forall t, u, v \in J$;

(c) $\mathfrak{u}_{0}=T, M \frac{T^{\alpha}}{\Gamma(\alpha+1)} \leq \mathfrak{u}_{0}, f(t, u, v) \geq 0, \forall t, u, v \in J$.

If

$$
\frac{(\widetilde{\mathrm{L}}+2) T^{\alpha} \ell}{\Gamma(\alpha+1)} \leq 1,
$$

then there exists at least one solution of problem (1) in $\mathrm{C}_{\mathrm{L}, \alpha}$ which can be approximated by the Krasnoselskij iteration

$$
u_{n+1}=(1-\lambda) u_{n}+\lambda u_{0}+\lambda \int_{0}^{t} \frac{(t-\tau)^{\alpha-1}}{\Gamma(\alpha)} f(\tau, u(\tau), u(u(\tau))) d \tau
$$

where $\lambda \in(0,1)$ and $u_{1} \in C_{L, \alpha}$ is arbitrary.

Proof. Consider the integral operator $\mathrm{P}: \mathrm{C}_{\mathrm{L}, \alpha} \rightarrow \mathrm{C}(\mathrm{J})$

$$
P u(t)=u_{0}+\int_{0}^{t} \frac{(t-\tau)^{\alpha-1}}{\Gamma(\alpha)} f(\tau, u(\tau), u(u(\tau))) d \tau, \quad t \in J, u \in C_{L, \alpha} .
$$


Our aim is show that $\mathrm{P}$ has a fixed point in $\mathrm{C}_{\mathrm{L}, \alpha}$. We proceed to apply Schauder fixed point theorem or Banach fixed point theorem.

First we show that $C_{L, \alpha}$ is invariant set with respect to $P$, i.e. $T\left(C_{L, \alpha}\right) \subset C_{L, \alpha}$. In virtue of condition (A4a) and for all $\mathrm{t} \in \mathrm{J}, \boldsymbol{u} \in \mathrm{C}_{\mathrm{L}, \alpha}$ we have

$$
\begin{aligned}
|P u(t)| & \leq\left|u_{0}\right|+\left|\int_{0}^{t} \frac{(t-\tau)^{\alpha-1}}{\Gamma(\alpha)} f(\tau, u(\tau), \mathfrak{u}(u(\tau))) d \tau\right| \\
& \leq\left|\mathfrak{u}_{0}\right|+M \frac{T^{\alpha}}{\Gamma(\alpha+1)} \leq T
\end{aligned}
$$

and

$$
\begin{aligned}
|\operatorname{Pu}(t)| & \geq\left|\mathfrak{u}_{0}\right|-\left|\int_{0}^{t} \frac{(t-\tau)^{\alpha-1}}{\Gamma(\alpha)} f(\tau, \mathfrak{u}(\tau), \mathfrak{u}(u(\tau))) d \tau\right| \\
& \geq\left|u_{0}\right|-M \frac{T^{\alpha}}{\Gamma(\alpha+1)} \geq \mathfrak{u}_{0}-M_{\mathfrak{u}_{0}} \geq 0 .
\end{aligned}
$$

Thus $(\mathrm{Pu})(\mathrm{t}) \in \mathrm{J}, \mathrm{t} \in \mathrm{J}$. In the similar manner of $(\mathrm{A} 4 \mathrm{a})$, we treat the cases $(\mathrm{A} 4 \mathrm{~b})$ and $(\mathrm{A} 4 \mathrm{c})$. Now for every $t_{1}, t_{2} \in J$, by (A3), we obtain

$$
\begin{aligned}
\left|(P u)\left(t_{1}\right)-(P u)\left(t_{2}\right)\right| & =\left|\int_{0}^{t_{1}} \frac{(t-\tau)^{\alpha-1}}{\Gamma(\alpha)} f(\tau, u(\tau), u(u(\tau))) d \tau-\int_{0}^{t_{2}} \frac{(t-\tau)^{\alpha-1}}{\Gamma(\alpha)} f(\tau, u(\tau), u(u(\tau))) d \tau\right| \\
& \leq M \frac{\left|t_{1}^{\alpha}-t_{2}^{\alpha}+2\left(t_{1}-t_{2}\right)^{\alpha}\right|}{\Gamma(\alpha+1)} \\
& \leq 2 M \frac{\left|t_{1}-t_{2}\right|^{\alpha}}{\Gamma(\alpha+1)} \\
& \leq L \frac{\left|t_{1}-t_{2}\right|^{\alpha}}{\Gamma(\alpha+1)} .
\end{aligned}
$$

Hence $(\mathrm{Pu}) \in \mathrm{C}_{\mathrm{L}, \alpha}$ whenever $\mathrm{u} \in \mathrm{C}_{\mathrm{L}, \alpha}$. Therefore, $\mathrm{P}: \mathrm{C}_{\mathrm{L}, \alpha} \rightarrow \mathrm{C}_{\mathrm{L}, \alpha}$ (i. e., $\mathrm{P}$ is a self-mapping of $\mathrm{C}_{\mathrm{L}, \alpha}$ ). Let $u, v \in \mathrm{C}_{\mathrm{L}, \alpha}$ and $\mathrm{t} \in \mathrm{J}$, by employing (A2) we have 


$$
\begin{aligned}
|(P u)(t)-(P v)(t)| & =\left|\int_{0}^{t} \frac{(t-\tau)^{\alpha-1}}{\Gamma(\alpha)} f(\tau, u(\tau), u(u(\tau))) d \tau-\int_{0}^{t} \frac{(t-\tau)^{\alpha-1}}{\Gamma(\alpha)} f(\tau, v(\tau), v(v(\tau))) d \tau\right| \\
& \leq \int_{0}^{t} \frac{(t-\tau)^{\alpha-1}}{\Gamma(\alpha)}|f(\tau, u(\tau), u(u(\tau)))-f(\tau, v(\tau), v(v(\tau)))| d \tau \\
& \leq \int_{0}^{t} \frac{(t-\tau)^{\alpha-1}}{\Gamma(\alpha)}[|u(\tau)-v(\tau)|+|u(u(\tau))-v(v(\tau))|] d \tau \\
& \leq \int_{0}^{t} \frac{(t-\tau)^{\alpha-1}}{\Gamma(\alpha)} \max [|u(\tau)-v(\tau)|+|u(u(\tau))-u(v(\tau))+u(v(\tau))-v(v(t))|] d \tau \\
& \leq \frac{T^{\alpha} \ell}{\Gamma(\alpha+1)}[\widetilde{\mathrm{L}}\|\mathfrak{u}-v\|+2\|\mathfrak{u}-v\|] \\
& \leq \frac{(\widetilde{L}+2) T^{\alpha} \ell}{\Gamma(\alpha+1)}\|\mathfrak{u}-v\|,
\end{aligned}
$$

where $1 \leq\|u-v\| \leq \mathrm{T}$ and

$$
\widetilde{\mathrm{L}}:=\max \frac{\mathrm{L}}{\Gamma(\alpha+1)}, \quad \alpha \in(0,1] .
$$

Now, by taking the supremum in the last assertion, we get

$$
\| \mathrm{Pu})-(\mathrm{P} v)\left\|\leq \frac{(\widetilde{\mathrm{L}}+2) \mathrm{T}^{\alpha} \ell}{\Gamma(\alpha+1)}\right\| \mathrm{u}-v \|
$$

If $\frac{(\widetilde{\mathrm{L}}+2) T^{\alpha} \ell}{\Gamma(\alpha+1)}<1$, then $\mathrm{P}$ is a contraction mapping and hence in view of Banach fixed point theorem, Eq. (1) has a unique solution. Now if

$$
\frac{(\widetilde{\mathrm{L}}+2) T^{\alpha} \ell}{\Gamma(\alpha+1)}=1
$$

then $\mathrm{P}$ is non-expansive and, hence, continuous; thus Schauder fixed point theorem implies that Eq. (1) has a solution in $C_{L, \alpha}$. Finally, in view of Lemmas 2.1 and 2.2, we obtain the second part of the theorem.

Next we establish the solution of Eq. (1) in a subset of $C_{L, \alpha}$ defined by

$$
\mathrm{C}_{\mathrm{L}, \alpha, \delta}=\left\{u \in \mathrm{C}_{\mathrm{L}, \alpha}: \mathrm{u}(\mathrm{t}) \leq \frac{\delta \mathrm{t}^{\alpha}}{\Gamma(\alpha+1)}, \forall \mathrm{t} \in \mathrm{J}\right\}, \quad \delta \in(0,1) .
$$

It is clear that $C_{L, \alpha, \delta}$ is non-empty, convex and compact subset in $\mathrm{C}[\mathrm{J}]$.

Theorem 3.2. Assume that the following conditions are satisfied:

$(\mathrm{A} 5) \mathrm{u}_{0} \leq \frac{\delta \mathrm{t}_{0}^{\alpha}}{2 \Gamma(\alpha+1)} \quad \mathrm{t}_{0}(\neq 0) \in \mathrm{J}$; 
(A6) If $L$ is the Lipschitz constant such that $\left|u\left(t_{1}\right)-u\left(t_{2}\right)\right| \leq \frac{L}{\Gamma(\alpha+1)}\left|t_{1}-t_{2}\right|^{\alpha}$, then $M \leq \min \left\{\frac{\delta}{2}, \frac{L}{2}\right\}$;

(A7) There exists a $\bar{\tau}>0$ such that $\bar{\tau}>-\frac{\ln (1-\delta)}{\delta\left(T-t_{0}\right)}, T \neq t_{0}$ and

$$
\frac{T^{\alpha-1} \ell}{\Gamma(\alpha) \bar{\tau}}\left(\frac{1}{\delta}+\widetilde{\mathrm{L}}+1\right) \max \left\{e^{\bar{\tau} \mathrm{t}_{0}}-1,1-e^{\bar{\tau}\left(\mathrm{t}_{0}-\mathrm{T}\right)}\right\} \leq 1
$$

If (A2), (A4) hold then there exists at least one solution of problem (1) in $C_{L, \alpha, \delta}$ which can be approximated by the Krasnoselskij iteration

$$
u_{n+1}=(1-\lambda) u_{n}+\lambda u_{0}+\lambda \int_{0}^{t} \frac{(t-\tau)^{\alpha-1}}{\Gamma(\alpha)} f(\tau, u(\tau), u(u(\tau))) d \tau
$$

where $\lambda \in(0,1)$ and $u_{1} \in C_{L, \alpha, \delta}$ is arbitrary.

Proof. We assume the Banach space C[J] endowed with Bieleckis norm given by the formula

$$
\begin{gathered}
\|u\|_{B}=\max _{t \in J}|u(t)| e^{-s\left(t-t_{0}\right)}, \quad s>0, t>t_{0} \\
\left(t, s, t_{0} \in J=[0, T], T<\infty\right) .
\end{gathered}
$$

Let $\mathrm{P}$ be defined as in the proof of Theorem 3.1. By assumptions (A2), (A4), and (A6), it follows that

$$
\mathrm{P}\left(\mathrm{C}_{\mathrm{L}, \alpha, \delta}\right) \subset \mathrm{C}_{\mathrm{L}, \alpha, \delta}
$$

Now we prove that $C_{L, \alpha, \delta}$ is an invariant set with respect to the operator $P$. Indeed, if $u \in C_{L, \alpha, \delta}$ and $\mathrm{t} \in \mathrm{J}$ then in view of (A5) and (A6), we have

$$
\begin{aligned}
\mathrm{Pu}(\mathrm{t}) & \leq \mathrm{u}_{0}+M \frac{\mathrm{t}^{\alpha}}{\Gamma(\alpha+1)} \\
& =\mathrm{u}_{0}+M \frac{\left(\mathrm{t}^{\alpha}-\mathrm{t}_{0}^{\alpha}\right)+\mathrm{t}_{0}^{\alpha}}{\Gamma(\alpha+1)} \\
& \leq \frac{\delta \mathrm{t}_{0}^{\alpha}}{2 \Gamma(\alpha+1)}+\frac{\delta \mathrm{t}^{\alpha}}{2 \Gamma(\alpha+1)}-\frac{\delta \mathrm{t}_{0}^{\alpha}}{2 \Gamma(\alpha+1)}+\frac{\delta \mathrm{t}_{0}^{\alpha}}{2 \Gamma(\alpha+1)} \\
& \leq \frac{\delta \mathrm{t}^{\alpha}}{\Gamma(\alpha+1)}, \quad \mathrm{t}>\mathrm{t}_{0},
\end{aligned}
$$

that is $\mathrm{Pu} \in \mathrm{C}_{\mathrm{L}, \alpha, \delta}$.

Let $u, v \in \mathrm{C}_{\mathrm{L}, \alpha, \delta}$ and $\mathrm{t} \in \mathrm{J}$, we have 


$$
\begin{aligned}
|(P u)(t)-(P v)(t)| & =\left|\int_{0}^{t} \frac{(t-\tau)^{\alpha-1}}{\Gamma(\alpha)} f(\tau, u(\tau), u(u(\tau))) d \tau-\int_{0}^{t} \frac{(t-\tau)^{\alpha-1}}{\Gamma(\alpha)} f(\tau, v(\tau), v(v(\tau))) d \tau\right| \\
& \leq \frac{T^{\alpha-1} \ell}{\Gamma(\alpha)}\left|\int_{0}^{t}(|u(\tau)-v(\tau)|+\widetilde{L}|u(\tau)-v(\tau)|+|u(v(\tau))-v(v(\tau))|) d \tau\right| \\
& \leq \frac{T^{\alpha-1} \ell}{\Gamma(\alpha)}\left(\left|\int_{0}^{t}(\widetilde{L}+1) e^{s\left(\tau-t_{0}\right)} d \tau\right|+\left|\int_{0}^{t} e^{s\left(\delta \tau-t_{0}\right)} d \tau\right|\right)\|u-v\|_{B} \\
& \leq \frac{T^{\alpha-1} \ell}{\Gamma(\alpha)}\left(\left|\frac{(\widetilde{L}+1)}{s}\left(e^{s\left(t-t_{0}\right)}-1\right)\right|+\frac{1}{\delta s}\left|e^{s\left(\delta t-t_{0}\right)}-e^{s\left(\delta t_{0}-t_{0}\right)}\right|\right)\|u-v\|_{B} .
\end{aligned}
$$

This yields

$$
\begin{aligned}
|(\mathrm{Pu})(\mathrm{t})-(\mathrm{P} v)(\mathrm{t})| e^{-s\left(\tau-\mathrm{t}_{0}\right)} & \leq \frac{T^{\alpha-1} \ell}{s \Gamma(\alpha)}\left((\widetilde{\mathrm{L}}+1)\left|1-e^{-s\left(\mathrm{t}-\mathrm{t}_{0}\right)}\right|+\frac{1}{\delta}\left|e^{\mathrm{s}(\delta-1) \mathrm{t}}-e^{\mathrm{s}\left(\delta \mathrm{t}_{0}-\mathrm{t}\right)}\right|\right)\|\mathrm{u}-v\|_{\mathrm{B}} \\
& :=\mathrm{L}(\mathrm{t})\|\mathrm{u}-v\|_{\mathrm{B}}
\end{aligned}
$$

where $L(t)$ is a continuous function. Then there exists a constant $\widehat{L}>0$ such that

$$
\max _{\mathrm{t} \in \mathrm{J}}|\mathrm{L}(\mathrm{t})| \leq \widehat{\mathrm{L}}
$$

Thus we have

$$
\|\mathrm{Pu}-\mathrm{P} v\|_{\mathrm{B}} \leq \widehat{\mathrm{L}}\|\mathrm{u}-v\|_{\mathrm{B}},
$$

which shows that $\mathrm{P}$ is Lipschitzian, hence continuous. By Schauders fixed point theorem it follows that $\mathrm{T}$ has at least one fixed point which is actually a solution of the initial value problem (1).

We proceed to show that $\mathrm{P}$ is non-expansive function. The function

$$
g(t)=1-e^{-s\left(t-t_{0}\right)}, \quad s>0, t>t_{0}
$$

is strictly increasing on $\mathrm{J}$ and $\mathrm{g}\left(\mathrm{t}_{\mathrm{o}}\right)=0$; furthermore,

$$
\max _{t \in J} g(t)=\max \left\{e^{\widetilde{\tau} t_{0}}-1,1-e^{\widehat{\tau}\left(t_{0}-T\right)}\right\} .
$$

Similarly for the function

$$
h(t)=e^{s(\delta-1) t}-e^{s\left(\delta t_{0}-t\right)}
$$

then

$$
h^{\prime}(t)=s e^{s(\delta-1) t}\left[(\delta-1)+e^{s \delta\left(t-t_{0}\right)}\right]
$$

Now the function

$$
k(t)=(\delta-1)+e^{s \delta\left(t-t_{0}\right)}
$$


is strictly decreasing on J; hence,

$$
k(t) \geq k(T)=(\delta-1)+e^{s \delta\left(T-t_{0}\right)} .
$$

For $\delta \in(0,1)$ and $\mathrm{T} \neq \mathrm{t}_{0}$ then by the assumption (A7) there exists a $\bar{\tau}>0$ such that

$$
\bar{\tau}>-\frac{\ln (1-\delta)}{\delta\left(T-t_{0}\right)}, T \neq t_{0}
$$

which implies that $k(T)>0$ and hence $h$ is strictly increasing on J. If we put $s=\bar{\tau}$ we have

$$
\max _{t \in J}|h(t)|=\max \left\{\left|1-e^{s \delta t_{0}}\right|,\left|e^{s(\delta-1) T}-e^{s\left(\delta t_{0}-T\right)}\right|\right\} .
$$

But since $\delta \in(0,1)$ thus we get

$$
\begin{aligned}
\left|e^{s(\delta-1) T}-e^{s\left(\delta t_{0}-T\right)}\right| & =e^{s(\delta-1) T}\left|1-e^{s \delta\left(t_{0}-T\right)}\right| \\
& \leq 1-e^{s \delta\left(t_{0}-T\right)}
\end{aligned}
$$

for sufficient $s, \delta, T$ and $t_{0}$. Moreover, we have

$$
\left|1-e^{s \delta t_{0}}\right| \leq e^{s \delta t_{0}}-1
$$

Consequently, we receive

$$
\mathrm{L}(\mathrm{t}) \leq \max \left\{e^{s \delta \mathrm{t}_{0}}-1,1-e^{\mathrm{s} \delta\left(\mathrm{t}_{0}-\mathrm{T}\right)}\right\} \frac{\mathrm{T}^{\alpha-1} \ell}{s \Gamma(\alpha)}\left(\frac{1}{\delta}+\widetilde{\mathrm{L}}+1\right) .
$$

This shows that $\mathrm{P}$ is non-expansive.

Similar argument holds when $T=t_{0}$ in Eq. (4) we have $k(T)=\delta>0$ hence $h$ is strictly increasing on J. Finally, one can use Lemmas 2.1 and 2.2 to obtain the second part of the theorem. This completes the proof.

Example 3.1. Consider the following initial value problem associated to an fractional iterative differential equation

$$
\left\{\begin{array}{l}
D^{0.5} u(t)=-\frac{1}{3}+\frac{1}{4} u(t)+\frac{1}{4} u(u(t)), \quad t \in[0,1] \\
u(0)=\frac{1}{3}
\end{array}\right.
$$

where $u \in C^{1}([0,1],[0,1])$. We are focused in the solutions $u \in C^{1}([0,1],[0,1])$ belonging to the set

$$
\begin{aligned}
C_{1,0.5} & =\left\{u:\left|u\left(t_{1}\right)-u\left(t_{2}\right)\right| \leq \frac{1}{\Gamma\left(\frac{3}{2}\right)}\left|t_{1}-t_{2}\right|^{0.5}, \forall t_{1}, t_{2} \in[0,1]\right\} \\
& =\left\{u:\left|u\left(t_{1}\right)-u\left(t_{2}\right)\right| \leq \frac{1}{0.886} \sqrt{\left|t_{1}-t_{2}\right|}, \forall t_{1}, t_{2} \in[0,1]\right\} \\
& =\left\{u:\left|u\left(t_{1}\right)-u\left(t_{2}\right)\right| \leq 1.1 \sqrt{\left|t_{1}-t_{2}\right|}, \forall t_{1}, t_{2} \in[0,1]\right\} .
\end{aligned}
$$


To satisfy (A4a), we have $M \leq \frac{\mathrm{L}}{2} \simeq \frac{1}{2}, M_{\frac{1}{3}}=\max \left\{\frac{1}{3}, \frac{2}{3}\right\}=\frac{2}{3}=0.666$ and

$$
M \frac{T^{\alpha}}{\Gamma(\alpha+1)}=\frac{1}{2} \times \frac{1}{0.886}=0.56<0.666
$$

Hence (A4a) is satisfied. The function $f(t)=-\frac{1}{3}+\frac{1}{4}(u+v), v:=u(u(t))$, is Lipschitzian with the Lipschitz constant $\ell=\frac{1}{4}$. This shows that

$$
\frac{(\widetilde{\mathrm{L}}+2) \top^{\alpha} \ell}{\Gamma(\alpha+1)}=\frac{3.1 \times 0.25}{0.886}=0.874<1 .
$$

Therefore, by Theorem 3.1 we obtain information on the existence and approximation of the solutions of the initial value problem (5).

If we consider the function $f(t)=-\frac{1}{3}+\frac{286}{1000}(u+v)$ in Example 3.1, then we obtain

$$
\frac{(\widetilde{\mathrm{L}}+2) \mathrm{T}^{\alpha} \ell}{\Gamma(\alpha+1)}=\frac{3.1 \times 0.286}{0.886} \simeq 1
$$

Therefore, again by Theorem 3.1 we pose the existence and approximation of the solutions of the initial value problem (5).

Again, we consider the problem (5) on the interval $\left[\frac{3}{4}, 1\right]$ for $\ell=0.015$, where $u \in C^{1}\left(\left[\frac{3}{4}, 1\right],\left[\frac{3}{4}, 1\right]\right)$. We are interested in the solutions $u \in C^{1}\left(\left[\frac{3}{4}, 1\right],\left[\frac{3}{4}, 1\right]\right)$ belonging to the set

$$
\begin{aligned}
C_{1, \frac{1}{2}, \frac{3}{4}} & \left.=\left\{u \in C_{1, \frac{1}{2}}: u(t) \leq \frac{\delta t^{\alpha}}{\Gamma(\alpha+1)}, \forall t \in J\right\}, \quad \delta \in(0,1)\right\} \\
& =\left\{u: u(t) \leq \frac{\frac{3}{4} t^{\frac{1}{2}}}{\Gamma\left(\frac{3}{2}\right)}, \forall t \in\left[\frac{3}{4}, 1\right]\right\} \\
& =\left\{u: u(t) \leq 0.846 \sqrt{t}, t \in\left[\frac{3}{4}, 1\right]\right\} .
\end{aligned}
$$

Our aim is to satisfy the assumptions of Theorem 3.2. (A2) and (A4) are valid. Since $\mathfrak{u}_{0}=\frac{1}{3}$ and $t_{0}=\frac{3}{4}$ we have

$$
u_{0} \leq \frac{\delta t_{0}^{\alpha}}{2 \Gamma(\alpha+1)} \Longrightarrow \frac{1}{3}<\frac{3}{8}
$$

hence (A5) is satisfied. Moreover, a computation gives

$$
M \leq \min \left\{\frac{\delta}{2}, \frac{L}{2}\right\}=\min \left\{\frac{\delta}{2}, \frac{L}{2}\right\}=\left\{\frac{3}{8}, \frac{1}{2}\right\}=\frac{3}{8}
$$

thus (A6) is satisfied. Now we proceed to satisfy (A7); since

$$
-\frac{\ln (1-\delta)}{\delta\left(T-t_{0}\right)}=\frac{-\ln \frac{1}{4} \times 16}{3}=6.933
$$


and

$$
\max \left\{e^{\bar{\tau} t_{0}}-1,1-e^{\bar{\tau}\left(t_{0}-T\right)}\right\}=\max \{189.5, .826\}
$$

then for $\bar{\tau}=7$ we impose

$$
\begin{aligned}
\frac{\mathrm{T}^{\alpha-1} \ell}{\Gamma(\alpha) \bar{\tau}}\left(\frac{1}{\delta}+\widetilde{\mathrm{L}}+1\right) \max \left\{e^{\bar{\tau} \mathrm{t}_{0}}-1,1-e^{\bar{\tau}\left(\mathrm{t}_{\mathrm{o}}-\mathrm{T}\right)}\right\} & =\frac{0.15}{37.17} \max \{189.5, .826\} \\
& =0.758<1 .
\end{aligned}
$$

Hence in view of Theorem 3.2, problem (5) has a solution in the set $C_{1, \frac{1}{2}, \frac{3}{4}}$. Note that when $\ell=0.025$ we obtain

$$
\begin{aligned}
\frac{\mathrm{T}^{\alpha-1} \ell}{\Gamma(\alpha) \bar{\tau}}\left(\frac{1}{\delta}+\widetilde{\mathrm{L}}+1\right) \max \left\{e^{\bar{\tau} \mathrm{t}_{0}}-1,1-e^{\bar{\tau}\left(\mathrm{t}_{\mathrm{o}}-\mathrm{T}\right)}\right\} & =\frac{0.25}{37.17} \max \{189.5, .826\} \\
& =1.137>1 .
\end{aligned}
$$

Thus problem (5) hasn't a solution in $C_{1, \frac{1}{2}, \frac{3}{4}}$. While, for $\ell \simeq .02$, implies

$$
\frac{T^{\alpha-1} \ell}{\Gamma(\alpha) \bar{\tau}}\left(\frac{1}{\delta}+\widetilde{\mathrm{L}}+1\right) \max \left\{e^{\bar{\tau} \mathrm{t}_{0}}-1,1-e^{\bar{\tau}\left(\mathrm{t}_{0}-\mathrm{T}\right)}\right\} \simeq 1
$$

therefore, in virtue of Theorem 3.2, Eq.(5) has a solution.

Moreover, we can observe that problem (5) hasn't a solution on the set $C_{1, \frac{1}{2}, \frac{1}{2}}$ over the interval $\left[\frac{1}{2}, 1\right]$ :

$$
\begin{aligned}
C_{1, \frac{1}{2}, \frac{1}{2}} & \left.=\left\{u \in C_{1, \frac{1}{2}}: u(t) \leq \frac{\delta t^{\alpha}}{\Gamma(\alpha+1)}, \forall t \in J\right\}, \quad \delta \in(0,1)\right\} \\
& =\left\{u: u(t) \leq \frac{\frac{1}{2} t^{\frac{1}{2}}}{\Gamma\left(\frac{3}{2}\right)}, \forall t \in\left[\frac{1}{2}, 1\right]\right\} \\
& =\left\{u: u(t) \leq 0.564 \sqrt{t}, t \in\left[\frac{1}{2}, 1\right]\right\} .
\end{aligned}
$$

For $\mathfrak{u}_{0}=\frac{1}{3}, t_{0}=\frac{1}{2}, \alpha=\frac{1}{2}, \delta=\frac{1}{2}$, a calculation poses

$$
\mathrm{u}_{0} \leq \frac{\delta \mathrm{t}_{0}^{\alpha}}{2 \Gamma(\alpha+1)} \Longrightarrow \frac{1}{3}>\frac{0.35}{1.772}
$$

therefore, condition (A5) dose not satisfy.

Finally, problem (5) hasn't a solution on the set $C_{1, \frac{1}{2}, \frac{1}{2}}$ over the interval $\left[\frac{3}{4}, 1\right]$ :

$$
\begin{aligned}
C_{1, \frac{1}{2}, \frac{1}{2}} & \left.=\left\{u \in C_{1, \frac{1}{2}}: u(t) \leq \frac{\delta t^{\alpha}}{\Gamma(\alpha+1)}, \forall t \in J\right\}, \quad \delta \in(0,1)\right\} \\
& =\left\{u: u(t) \leq \frac{\frac{1}{2} t^{\frac{1}{2}}}{\Gamma\left(\frac{3}{2}\right)}, \forall t \in\left[\frac{3}{4}, 1\right]\right\} \\
& =\left\{u: u(t) \leq 0.5 \sqrt{t}, t \in\left[\frac{3}{4}, 1\right]\right\} .
\end{aligned}
$$


For $\mathrm{u}_{0}=\frac{1}{3}, \mathrm{t}_{0}=\frac{3}{4}, \alpha=\frac{1}{2}, \delta=\frac{1}{2}$, a calculation yields

$$
\mathrm{u}_{0} \leq \frac{\delta \mathrm{t}_{0}^{\alpha}}{2 \Gamma(\alpha+1)} \Longrightarrow \frac{1}{3}>\frac{1}{4}
$$

therefore, condition (A5) dose not satisfy.

As such iterative fractional differential equations are used to generalize the model infective disease processes, pattern formation in the plane, and are important in investigations of dynamical systems, future works will be also devoted to them.

Received: November 2011. Revised: August 2012.

\section{References}

[1] R. W. Ibrahim, S. Momani, On the existence and uniqueness of solutions of a class of fractional differential equations, J. Math. Anal. Appl. 334 (2007) 1-10.

[2] S. M. Momani, R. W. Ibrahim, On a fractional integral equation of periodic functions involving Weyl-Riesz operator in Banach algebras, J. Math. Anal. Appl. 339 (2008) 1210-1219.

[3] R. A. El-Nabulsi, The fractional calculus of variations from extended Erdelyi-Kober operatorInt. J. Mod. Phys. B23(16)(2009) 3349-3361.

[4] Z. Wei, W. Dong, J. Che, Periodic boundary value problems for fractional differential equations involving Riemann-Liouville fractional derivative, Nonlinear Analysis: Theory, Methods and Applications, 73(10) (2010) 3232-3238.

[5] B. Ahmad, S. K. Ntouyas, A. Alsaedi, New existence results for nonlinear fractional differential equations with three-point integral boundary conditions, Advances in Difference Equations, Volume 2011, Article ID 107384, 11 pages.

[6] J. Sabatier, O. P. Agrawal, J. A. Tenreiro Machado, Advance in Fractional Calculus: Theoretical Developments and Applications in Physics and Engineering, Springer, 2007.

[7] I. Podlubny, Fractional Differential Equations, Acad. Press, London, 1999.

[8] A. A. Kilbas, H. M. Srivastava and J.J. Trujillo, Theory and applications of fractional differential equations. North-Holland, Mathematics Studies, Elsevier 2006.

[9] R. W. Ibrahim, M. Darus, Subordination and superordination for analytic functions involving fractional integral operator, Complex Variables and Elliptic Equations, 53 (2008) 1021-1031.

[10] R. W. Ibrahim, M. Darus, Subordination and superordination for univalent solutions for fractional differential equations, J. Math. Anal. Appl. 345 (2008) 871-879. 
[11] R. W. Ibrahim, Existence and uniqueness of holomorphic solutions for fractional Cauchy problem, J. Math. Anal. Appl. 380 (2011) 232-240.

[12] V. Berinde, Iterative Approximation of Fixed Points,2nd Ed.,Springer Verlag, Berlin Heidelberg New York, 2007.

[13] M. Edelstein, A remark on a theorem of M. A. Krasnoselskij, Amer. Math. Monthly, 73(1966) 509-510.

[14] E. Egri, I. Rus, First order iterative functional-dierential equation with parameter, Stud. Univ. Babes-Bolyai Math. 52 (2007) 67-80.

[15] C. Chidume, Geometric Properties of Banach spaces and nonlinear Iterations, Springer Verlag, Berlin, Heidelberg, New York, 2009.

[16] Yang, D. and Zhang, W., Solution of equivariance for iterative differential equations, Appl. Math. Lett. 17(2004) 759-765.

[17] A. Ronto, M. Ronto, Succsesive approximation method for some linear boundary value problems for differential equations with a special type of argument deviation, Miskolc Math. Notes, 10(2009) 69-95.

[18] V. Berinde, Existence and approximation of solutions of some first order iterative differential equations, Miskolc Math. Notes,Vol. 11 (2010) pp. 1326. 